\title{
An effective structure prediction method for layered materials based on 2D particle swarm optimization algorithm
}

\author{
Yanchao Wang, ${ }^{1}$ Maosheng Miao,,${ }^{2,3}$ Jian Lv, ${ }^{1}$ Li Zhu, ${ }^{1}$ Ketao Yin, ${ }^{1}$ Hanyu Liu, ${ }^{1}$ \\ and Yanming $\mathrm{Ma}^{1, \mathrm{a})}$ \\ ${ }^{1}$ State Key Laboratory of Superhard Materials, Jilin University, Changchun 130012, China \\ ${ }^{2}$ Materials Reserch Laboratory, University of California Santa Barbara, Santa Barbara, \\ California 93110, USA \\ ${ }^{3}$ Beijing Computational Science Research Center, Beijing, 100084, People's Republic of China
}

(Received 27 August 2012; accepted 16 November 2012; published online 12 December 2012)

\begin{abstract}
A structure prediction method for layered materials based on two-dimensional (2D) particle swarm optimization algorithm is developed. The relaxation of atoms in the perpendicular direction within a given range is allowed. Additional techniques including structural similarity determination, symmetry constraint enforcement, and discretization of structure constructions based on space gridding are implemented and demonstrated to significantly improve the global structural search efficiency. Our method is successful in predicting the structures of known 2D materials, including single layer and multi-layer graphene, 2D boron nitride $(\mathrm{BN})$ compounds, and some quasi-2D group 6 metals(VIB) chalcogenides. Furthermore, by use of this method, we predict a new family of monolayered boron nitride structures with different chemical compositions. The first-principles electronic structure calculations reveal that the band gap of these N-rich BN systems can be tuned from $5.40 \mathrm{eV}$ to $2.20 \mathrm{eV}$ by adjusting the composition. () 2012 American Institute of Physics. [http://dx.doi.org/10.1063/1.4769731]
\end{abstract}

\section{INTRODUCTION}

Dimensionality, being one of the most important material parameters, can totally alter the structure of a compound at fixed composition and can therefore change its properties drastically. Thus, the structural prediction of different dimensions has lately become a hot issue. Of these different dimensional structures, two-dimensional (2D) materials are of special importance $e^{1,2}$ because they are usually parent structures of one-dimensional nanotubes and zero-dimensional nanocages. Furthermore, the unusual electronic and structural properties of 2D materials are very different from 3D materials and this has resulted in their being widely applied in electronics and optoelectronics devices. In particular, recent progress has both illustrated striking fundamental physics ${ }^{3}$ in such systems and also revealed potential applications of 2D materials in replacing $\mathrm{Si}$ technology for faster and smaller electronics devices in the future.

Since the properties are intimately tied to the atomic arrangement of materials, the determination, prediction and design of structures are key steps in engineering functional materials and studying their related physics. In the past decade, several methods ${ }^{4-13}$ have been developed to predict the structures of crystals and clusters. We recently realized the high efficiency of the particle swarm optimization (PSO) method in searching materials structures and have implemented it in the Crystal structure Analysis by Particle Swarm Optimization (CALYPSO) code for predicting crystal structure. ${ }^{14,15}$

\footnotetext{
a) Author to whom correspondence should be addressed. Electronic mail: mym@jlu.edu.cn, URL: http://mym.calypso.cn.
}

CALYPSO can efficiently explore the multidimensional potential energy surfaces of a periodic system at given external conditions (e.g., pressure) and requires no a priori structure information except the chemical compositions. The method has been successfully applied in predicting crystal structures for various high-pressure systems including semiconducting phase of lithium, ${ }^{16}$ the superconducting phases of $\mathrm{Bi}_{2} \mathrm{Te}_{3},{ }^{17}$ partially ionic ice, ${ }^{18}$ spiral chain $\mathrm{O} 4$ form of dense oxygen, ${ }^{19}$ sodalite-like clathrate calcium hybride,${ }^{20}$ atomic clusters, ${ }^{21}$ and the single layer 2D structures. ${ }^{22,23}$

The 2D materials are not necessarily planar. Their atomic layers may buckle in the perpendicular direction. In addition, many materials are quasi-2D in the sense that they consist of multiple layers and the atoms in neighboring layers form chemical bonds. In order to capture all these structural features of 2D and quasi-2D materials, we developed a new 2D structure search module in CALYPSO code that is based on 2D PSO algorithm, but allows the relaxation of atomic coordinate in the perpendicular direction. This new implementation is benchmarked for a number of selected $2 \mathrm{D}$ and quasi-2D materials featuring known layered structures, such as single layer graphene, $\mathrm{B}$ and $\mathrm{BN}$, multi-layer graphene, and some group VIB chalcogenides $\left(\mathrm{MoS}_{2}\right.$, etc.). Furthermore, by using the new method, we investigated the structures of compounds and found a strong correlation between the parity of the building rings of these compounds and their fundamental energy gap. These results suggest a way of controlling the electronic properties via the control of their composition in this new family of layered materials.

This paper is arranged as follows. In Sec. II, the method and implementation of the PSO algorithm is discussed in 
details. The results of test calculations on selected 2D and quasi-2D materials and the calculations on a new family of $B_{x} N_{y}$ compounds are presented in Sec. III. Finally, a summary is provided in Sec. IV.

\section{METHOD AND IMPLEMENTATION}

A 2D structure search module is implemented in CALYPSO code. Similar to 3D bulk structure search, it also consists of four steps as shown by the flow chart in Fig. 1. The module consists of three major components, including the random structure generation, the evolution of the structures based on PSO algorithm, and the local optimization of the structures.

New random structures need to be constructed for both the initial generation and the new generations of structures (1st and 4th steps). Additional techniques, including the symmetry constraints, similar structure elimination, and the space gridding method, are implemented in order to improve the efficiency of structure generation and to maximize the coverage of the configuration space with finite number of structures.

The construction of new structures starts from a random selection of a symmetry group from 17 plane space groups. The 2D lattice parameters including the length of two lattice vectors and the angle between them are randomly generated according to the symmetry and the confined area. The coordinates of the atoms are set with respect to the Wyckoff positions of the symmetry group. The symmetry group of every new structure is compared with the symmetry groups of existing structures, and the new structure is eliminated if its symmetry group represents more than a certain percentage (for example, $80 \%$ ) of the structures. This will make the constructed structures covering different regions of the search space and therefore ensure the better sampling. The explicit application of symmetric constraints leads to the significant reduction of the search space and the optimization variables, and thus greatly speeds up the global structure search.

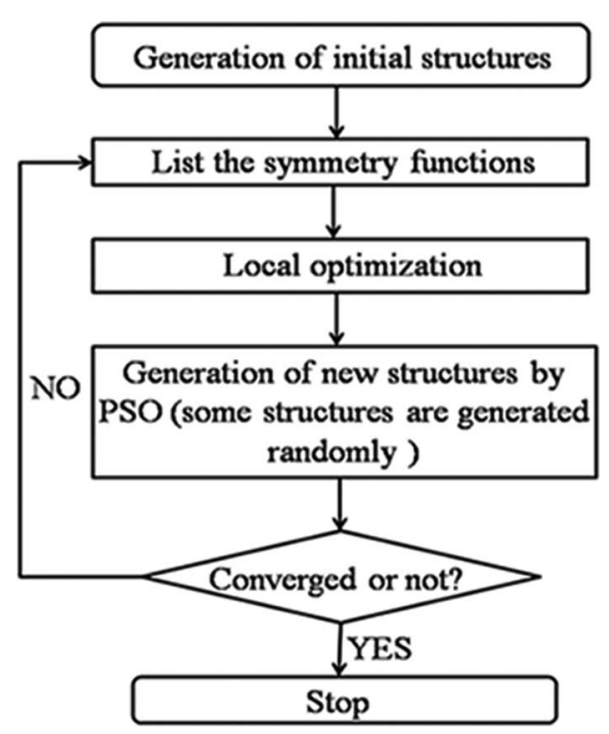

FIG. 1. The flow chart of the major steps in 2D structure search as implemented in CALYPSO code.
Another constraint to the structures is the minimal interatomic distances. ${ }^{24}$ Although the symmetry constraints can greatly reduce the search space, it is still enormous for large systems, especially while the number of atoms is larger than 20. In order to overcome this bottleneck and further reduce the search space, we implement an efficient method based on space gridding. Within this approach, a 2D grid points separated by the minimum atomic distances divide the unit cell of a structure. Only positions on the grid points are allowed for atoms. This approach will be evoked if the structure generation based on symmetry groups fails to produce structures that satisfy the minimum distance constraints in 50 attempts. The space gridding approach is more sufficient in enforcing the minimum distance constraints, although the symmetry information is no long reserved.

As shown before, ${ }^{24}$ the elimination of the similar structures can significantly improve the search efficiency. Yet, the determination of the similarity between two structures is not an easy task. In our earlier implementation, ${ }^{15}$ bond characterization matrix is used to identify structural similarity for the 3D structures. In this work, a set of simple multi-atom symmetry functions is used to quantify the structures. The detailed forms and the properties of the functions can be found in Ref. 25. These symmetry functions have been successfully applied to constructing the high-dimensional neural network potential. ${ }^{26,27}$ In our implementation, 33 symmetry functions with different parameters are used to represent atomic environment for each atom. A set of function values, which can be obtained by adding symmetry functions for each atom with the same parameters, is used to quantity the structures. The similarity between two structures is thus given by the difference of their symmetry functions.

All above techniques including symmetry constraints enforcement, discretization of structure constructions based on space gridding, and the constraint of the minimal inter-atomic distances are effective to reduce the search space. In order to examine the efficiency of our search space reduction techniques as implemented in CALYPSO code, the 2D system of boron with 8 atoms per simulation cell was used as test case. Around 100 structures were randomly generated and then optimized using the Vienna ab initio simulation package

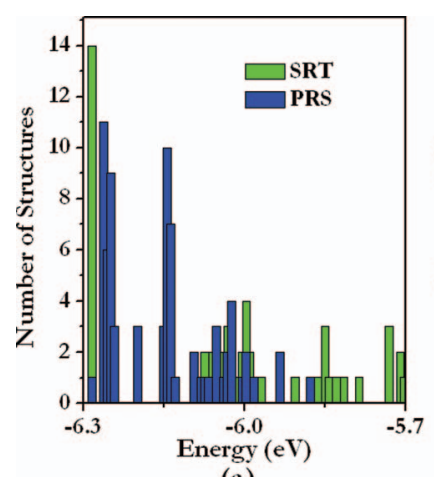

(a)

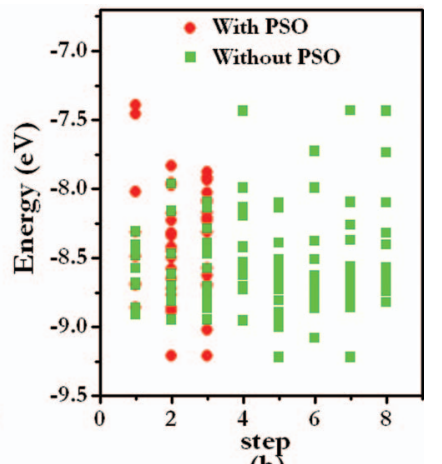

(b)
FIG. 2. (a) Energetic distributions of randomly generated structures with space reduction technique (SRT) and purely random search (PRS) for 2D boron (8 atoms per cell). (b) The history of a CALYPSO structure search performed on 2D carbon (16 atoms per cell) with PSO and without PSO. 
(VASP) code. Fig. 2(a) shows that the energetic distributions of these generated structures with and without space reduction technique, respectively. It is found that only one (1\%) global stable structure is generated if without search-space reduction technique. However, once the technique implemented in the generation of random structures, 14 (14\%) global stable structures were successfully produced. It clearly illustrates the importance of the search-space reduction technique in the generation of random structures for structure prediction. Furthermore, the structures generated by use of the search-space reduction distribute over a larger energy range, showing a higher structural diversity comparing with the random sampling. This is due to the fact that the constraints in the searchspace reduction greatly eliminate the duplicated and the similar structures being generated.

The evolution of the structures is performed by the PSO algorithm, ${ }^{28-30}$ which is inspired by the social behavior of birds flocking and designed to solve problems related to multidimensional optimization. Within this scheme, a structure in the search space is regarded as a particle. A set of particles (structures) is called a population or a generation. The positions of the particle are updated according to the following equation:

$$
x_{i, j}^{t+1}=x_{i, j}^{t}+v_{i, j}^{t+1},
$$

where $\mathrm{x}$ and $v$ are the position (consists of lattice parameters and atomic coordinates) and velocity, respectively ( $i$ is the atomic index, $j$ refers to the dimension of structure with $j$ $\in\{1,2\}$, and $t$ is the index of generation). The initial velocities are generated randomly. The new velocity of each particle is calculated from its previous unrelaxed atomic positions $x^{t}$, previous velocity $\mathrm{v}^{\mathrm{t}}$, the atomic positions of optimized structures (pbest), and the atomic positions of the structure with lowest energy in the entire population so far (gbest) by the following equation:

$v_{i, j}^{t+1}=\omega v_{i, j}^{t}+c_{1} r_{1}\left(\right.$ pbest $\left._{i, j}^{t}-x_{i, j}^{t}\right)+c_{2} r_{2}\left(\right.$ gbest $\left._{i, j}^{t}-x_{i, j}^{t}\right)$, where $\omega$ (in the range of $0.9 \sim 0.4$ ) denotes the inertia weight, $c_{1}=c_{2}=2, r_{1}$ and $r_{2}$ are two separately generated random numbers in the range $[0,1]$.

In order to examine the efficiency of PSO method in generating structures during the evolution, we compare the history profiles of CALYPSO runs with and without PSO for a 2D carbon unit with 16 atoms. The search-space reduction methods are employed for both PSO and non-PSO (random search) runs. Our results show that the inclusion of PSO in generating structures during evolution can reduce the number of generations of structure search from five in random search to two.

Following the initial and each PSO step, all the structures in one generation are relaxed with respect to their lattice parameters and atomic coordinates. Currently, CALYPSO code can interface with several external packages (e.g., VASP, ${ }^{31,32}$ SIESTA, ${ }^{33}$ Quantum Espresso, ${ }^{34}$ CASTEP, ${ }^{35,36}$ and GULP ${ }^{37}$ ) to perform the structural optimization. For a 2D system, all the structures are constrained in a plane. However, the atoms in layered materials may shift out of the plane, resulted in quasi2D structures. If the predicted structures are constrained in the plane, some of them may possess instability toward the perpendicular direction. In order to capture this feature of 2D materials, we allow the relaxation of the atoms in the perpendicular direction ( $z$ direction) within a certain range from $-\Delta z$ (for example, $0.1 \AA$ ) to $\Delta z$, so that the $2 \mathrm{D}$ nature of the structure is retained.

\section{TESTS AND APPLICATIONS}

We tested the 2D structure search module for a variety of 2D materials, including the known layered structures of $\mathrm{C}$, $\mathrm{BN}$, and B systems, the multi-layer graphene, and the 2D transition metal chalcogenides. Specifically, we studied the structural change of $\mathrm{B}_{\mathrm{x}} \mathrm{N}_{\mathrm{y}}$ system and examined the relation between their composition, structural topology, and electronic structure. The local optimization of structures and the calculation of their energies were done by density functional theory (DFT) as implemented in the Vienna ab initio simulation package (VASP). ${ }^{32}$ In the DFT plane-wave calculations, we used projector-augmented wave potentials $(\mathrm{PAW})^{38}$ to describe the core electrons and the generalized gradient approximation (GGA) of Perdew, Burke, and Ernzernhof (PBE) ${ }^{39}$ for exchange and correlation functional. All the atomic coordinates were relaxed until the Hellmann-Feynman forces were less than $0.01 \mathrm{eV} / \AA$. For the Brillouin zone integration, we generate $n \times m \times 1 k$-mesh in Monkhorst-Pack scheme, ${ }^{40}$ where $n$ and $m$ are determined by the lattice constant.

\section{A. Known single-layer systems}

We first applied our method to several known single layer systems including graphene, BN, and boron (Table I). Our method successfully found the most stable structure of 2D carbon, ${ }^{41}$ the hexagonal 2D-BN structure, ${ }^{42}$ and the known most stable $2 \mathrm{D} \alpha$-sheet boron ${ }^{43}$ within only one generation. These benchmarks suggest that our method is quite effective in predicting stable single-layer $2 \mathrm{D}$ materials. These structures are strictly $2 \mathrm{D}$ in the sense that all the atoms position in the same plane although the shift in perpendicular direction is allowed. This is not always the case. For example, a planar $\mathrm{B}_{3} \mathrm{C}$ structure [Fig. 3(a)] was predicted by a strict $2 \mathrm{D}$ structure search. ${ }^{22}$ However, the phonon modes with a large imaginary frequency at the zone center ( $\Gamma$ point) indicate instability at the perpendicular direction. Using our new implementation, a buckled layers structure [Fig. 3(b)] is predicted to be more favorable than the planar structure with an energy drop of $0.277 \mathrm{eV}$ (per formula unit).

\section{B. Non-stoichimetric B-N layers}

Using our method, the structures of single layer B-N system with various chemical compositions $\left(\mathrm{B}_{2} \mathrm{~N}_{3}, \mathrm{~B}_{3} \mathrm{~N}_{4}\right.$,

TABLE I. Testing results for the single layer systems including $\mathrm{C}, \mathrm{B}$, and $\mathrm{BN}$. The population sizes are chosen as 30 for $\mathrm{C}$ and $\mathrm{B}$ and 20 for $\mathrm{BN}$.

\begin{tabular}{lccc}
\hline \hline Systems & Structure & Generation & $N_{\text {pop }}$ \\
\hline $\mathrm{C}$ & Graphene & 1 & 30 \\
$\mathrm{~B}$ & $\alpha$-B & 1 & 30 \\
$\mathrm{BN}$ & $h$-BN & 1 & 20 \\
\hline \hline
\end{tabular}




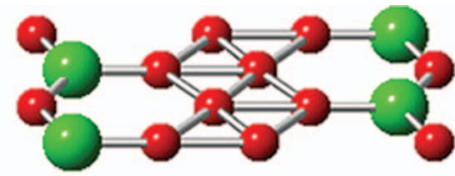

(a)

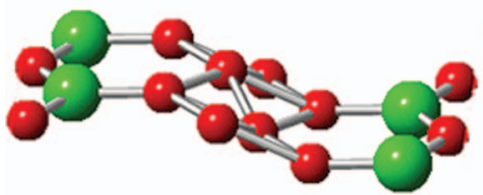

(b)

FIG. 3. The structures of single layer $\mathrm{B}_{3} \mathrm{C}$ as predicted by CALYPSO using (a) a strict 2D PSO structure search method, (b) current 2D PSO search that allows the relaxation of atoms in perpendicular direction. The larger green spheres and the smaller red spheres represent the $\mathrm{C}$ and the $\mathrm{B}$ atoms, respectively.

and $\mathrm{B}_{3} \mathrm{~N}_{5}$ ) were investigated. We found that the most stable 2D single layer structures of $\mathrm{B}_{2} \mathrm{~N}_{3}$ [Fig. 4(a)] and $\mathrm{B}_{3} \mathrm{~N}_{4}$ [Fig. 4(b)] consist of alternative arrays of B-N hexagons and arrays of B-N pentagon-octagon-pentagon (5-8-5), which share the similarity with the line defects in boron-nitride nano-sheets. ${ }^{44}$ Despite the composition, all the octagons consist of $5 \mathrm{~N}$ and $3 \mathrm{~B}$ atoms. All the pentagons in $\mathrm{B}_{3} \mathrm{~N}_{4}$ and half of the pentagons in $\mathrm{B}_{2} \mathrm{~N}_{3}$ consist of $3 \mathrm{~N}$ and $2 \mathrm{~B}$ atoms; whereas another half of the pentagons in $\mathrm{B}_{2} \mathrm{~N}_{3}$ consist of $4 \mathrm{~N}$ and $1 \mathrm{~B}$ atoms. All the hexagons consist of $3 \mathrm{~B}$ and $3 \mathrm{~N}$ atoms, except a quarter of them in $\mathrm{B}_{2} \mathrm{~N}_{3}$ consist of $2 \mathrm{~B}$ and $4 \mathrm{~N}$ atoms. On the other hand, PSO method predicts a novel structure for $\mathrm{B}_{3} \mathrm{~N}_{5}$, which is formed totally by the odd-numbered rings (pentagons and heptagons). This is the first time that an odd-numbered ring structure is found for a planar compound

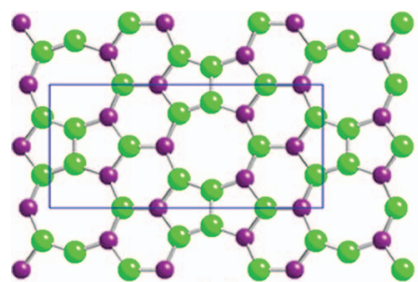

(a)

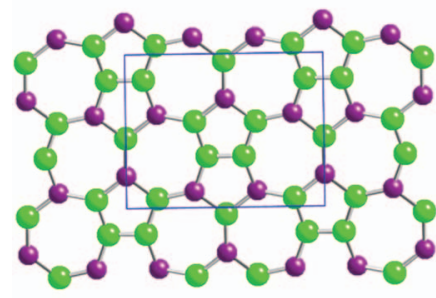

(c)

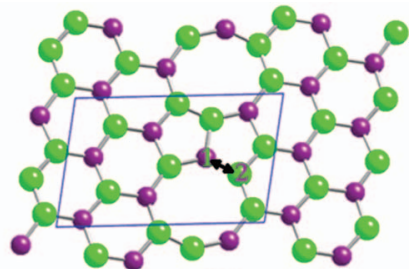

(b)

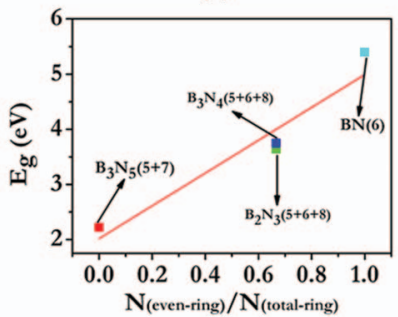

(d)
FIG. 4. The most stable single layer $2 \mathrm{D}$ structures of (a) $\mathrm{B}_{2} \mathrm{~N}_{3}$, (b) $\mathrm{B}_{3} \mathrm{~N}_{4}$, and (c) $\mathrm{B}_{3} \mathrm{~N}_{5}$, as predicted by PSO method. The green and the red balls show the positions of the $\mathrm{N}$ and $\mathrm{B}$ atoms, respectively. The $\mathrm{N}$ and the $\mathrm{B}$ atoms that can be switched to convert the structure into N-N arrays and B-N hexagons are marked by black arrows. (d) The relation of $2 \mathrm{D} \mathrm{B}_{\mathrm{x}} \mathrm{N}_{\mathrm{y}}$ band gap and the proportion of odd-numbered rings in total rings. The types of atomic rings in each compound are given in parenthesis and the label of $\mathrm{x}$-axis represents the proportion of the odd-membered rings relative to the total rings.
[Fig. 4(c)]. All the heptagons consist of $4 \mathrm{~N}$ and $3 \mathrm{~B}$; whereas, half hexagons consist of $3 \mathrm{~N}$ and $2 \mathrm{~B}$ and another half hexagons consist of $4 \mathrm{~N}$ and $1 \mathrm{~B}$.

For $\mathrm{B}_{2} \mathrm{~N}_{3}$, the structure can also be viewed as an array of $\mathrm{N}-\mathrm{N}$ dimmers inserted between the $\mathrm{BN}$ hexagons, which is the building unit of $h$-BN sheets (Fig. 4(a)) to form $[\mathrm{BN}]_{2}$ $\mathrm{N}_{2}-[\mathrm{BN}]_{2}$. Similarly, a structure (not the most stable one as predicted) of $\mathrm{B}_{3} \mathrm{~N}_{4}$ can be viewed as an array of N-N dimmers inserted between two $h$-BN double hexagonal arrays to form $[\mathrm{BN}]_{3}-\mathrm{N}_{2}-[\mathrm{BN}]_{3}$. Using the aforementioned $\mathrm{B}_{3} \mathrm{~N}_{4}$ structure as a prototype, the most stable $2 \mathrm{D}$ structure for $\mathrm{B}_{3} \mathrm{~N}_{4}$ can be obtained by switching the $\mathrm{N}$ and $\mathrm{B}$ atoms as marked in Fig. 4(b). It is important to note that the aforementioned structures of $\mathrm{B}_{2} \mathrm{~N}_{3}$ and $\mathrm{B}_{3} \mathrm{~N}_{4}$ have the similar structural topologies, namely, pentagonal-, hexagonal-, and octagonal-ring (5-6-8). They are very different to the topology of the stable structure of $\mathrm{B}_{3} \mathrm{~N}_{5}$ [Fig. 4(c)] that contains five- and seven-membered rings (5-7).

First-principles electronic structure calculations of these 2D BN structures show that these materials are semiconductors with a gap ranging from 1.30 to $4.61 \mathrm{eV}$, indicating their potential applications as electronic and optical materials. It is worth to notice that the local and the semi-local functionals can highly underestimate band gaps. Therefore, we also employ the screened hybrid functional of Heyd, Scuseria, and Ermzerhof ${ }^{45}$ (HSE) to examine the above gaps. Using this method, we obtained band gaps of 2.22, 3.64, 3.75, and $5.40 \mathrm{eV}$ for $\mathrm{B}_{3} \mathrm{~N}_{5}, \mathrm{~B}_{3} \mathrm{~N}_{4}, \mathrm{~B}_{2} \mathrm{~N}_{3}$, and $\mathrm{BN}$ [Fig. 4(d)]. The HSE gaps are about $0.9 \mathrm{eV}$ larger than the PBE values, however, the general trend of the band gap versus composition has not been changed for $\mathrm{B}_{\mathrm{x}} \mathrm{N}_{\mathrm{y}}$ system. Both PBE and HSE calculations show that the band gap decreases almost linearly with the increasing proportion of the odd-membered rings in these materials [Fig. 4(d)]. Thus, controlling the (x,y) composite of $\mathrm{B}_{\mathrm{x}} \mathrm{N}_{\mathrm{y}}$ and the synthesis conditions to produce the different structural topologies of 2D BN compounds can be used as an effective mean to tailor electronic properties.

\section{Multi-layer 2D systems}

The geometric structures of bilayers and trilayers of carbon were studied by our 2D structure search module. The electronic structure of multi-layer graphene strongly depends on the way of the stacking of the layers. ${ }^{46}$ The major challenge to structure search of this kind of system is that the energy differences among different stacking configurations are quite small because the interactions between the layers are exceedingly weak. As shown in Fig. 5, for bilayer graphene, there are two distinctive crystallographic stacking of the two graphene layers, ${ }^{47}$ including the graphite-like alternating Bernal stacking (AB $)^{48}$ [Fig. 5(a)] and the direct stacking of the graphene layers (AA) ${ }^{49}$ [Fig. 5(b)]. Other stackings as shown in Figs. 5(c) and 5(d), can be constructed from AA and $\mathrm{AB}$ stacking while shifting one layer horizontally. All these structures were found by the 2D module in CALYPSO without any a priori input. Furthermore, using the new 2D module, we also successfully found the two previously proposed stacking structures for trilayers carbon, including the ABA (Bernal) ${ }^{50}$ [Fig. 6(a)] and $\mathrm{ABC}^{51}$ (rhombohedral) 


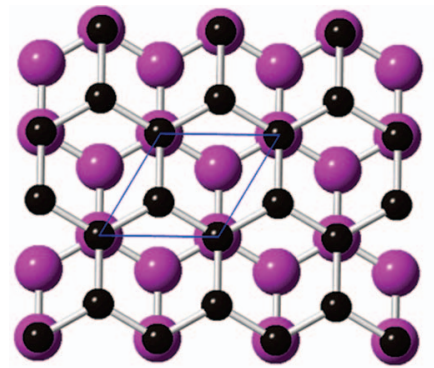

(a)

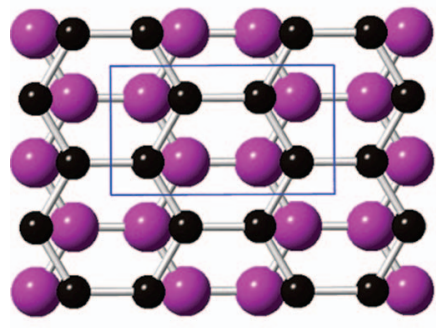

(c)

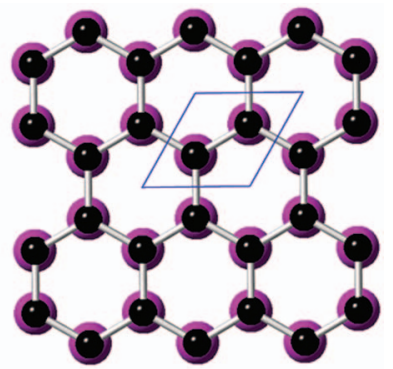

(b)

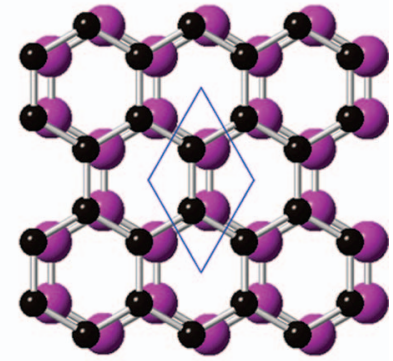

(d)
FIG. 5. The structures of bilayer graphene. The smaller black spheres show the carbon atoms in the top layer and the larger pink spheres show the carbon atoms in the bottom layer.

[Fig. 6(b)] stackings. Other stacking structures as shown in Figs. 6(c) and 6(d) are also found. Their energies are only slightly higher than those of the ABA and ABC stackings.

\section{Quasi-2D materials}

Although many 2D materials are planar, the majority of them are in quasi-2D structures, in which the atoms are in different layers and yet are chemically bonded with atoms in neighboring layers. For example, $\mathrm{MoS}_{2}$ is a major mineral of

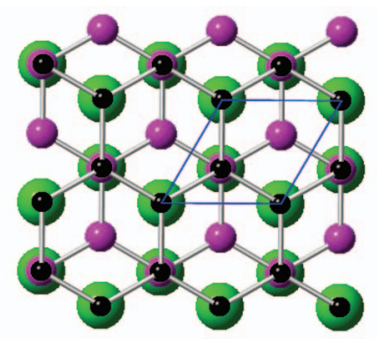

(a)

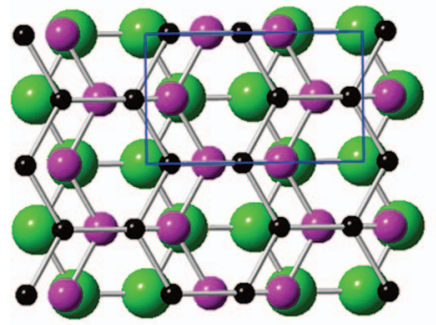

(c)

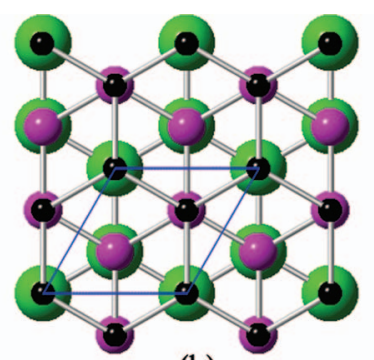

(b)

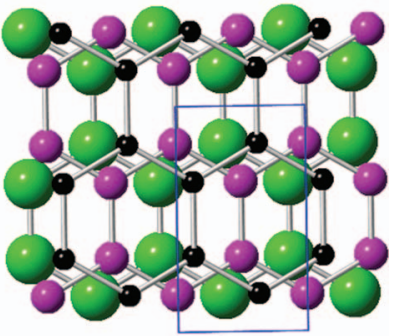

(d)
FIG. 6. The structures of trilayer graphene. The small black spheres, the pink spheres, and the large green spheres represent the carbon atoms in the top, the middle, and the bottom layers, respectively.

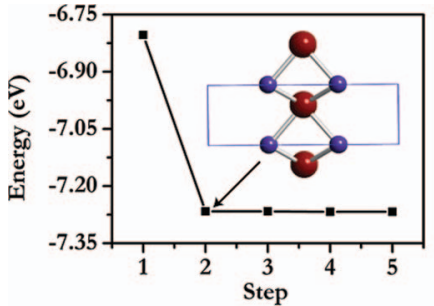

(a)

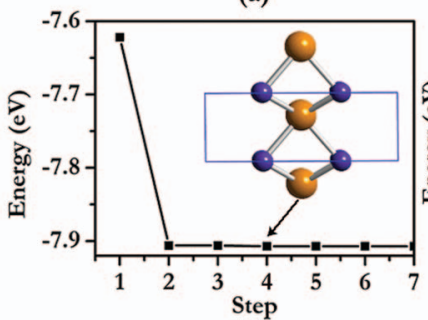

(c)

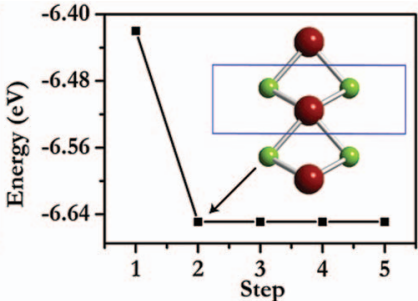

(b)

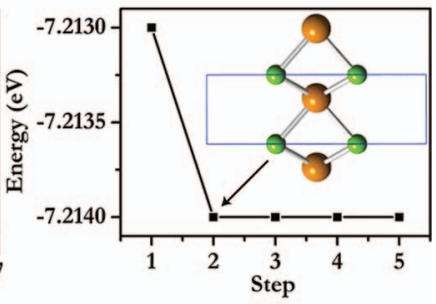

(d)
FIG. 7. The history of 2D PSO structure search for (a) $\mathrm{MoS}_{2}$, (b) $\mathrm{MoSe}_{2}$, (c) $\mathrm{WS}_{2}$, and (d) $\mathrm{WSe}_{2}$. The insets show the corresponding atomic structures. The larger spheres represent the group VIB elements Mo or W and the smaller spheres represent the chalcogenide elements S or Se.

Mo metal and is stable in a layered structure. In $\mathrm{MoS}_{2}$, each Mo atom bonds with 6 neighboring $\mathrm{S}$ atoms that form a trigonal prism. The prisms are interconnected by the shared $\mathrm{S}$ atoms and form a 2D layered structure, in which Mo layer is sandwiched by two S layers. ${ }^{52}$ As shown in Fig. 7(a), our 2D module finds this structure in two generations. The population size (the number of structures in each generation) is 20 , indicating the efficiency of our method in finding structures of quasi-2D materials. Similar structures are also found by our method for $\mathrm{MoSe}_{2}$ [Fig. 7(b)], ${ }^{52} \mathrm{WS}_{2}$ [Fig. 7(c)], and $\mathrm{WSe}_{2}$ [Fig. 7(d)], ${ }^{52}$ in either two, or four generations.

\section{CONCLUSION}

We have developed an efficient method based on the PSO algorithm for predicting the structures of 2D systems including single- and multi-layers structures and quasi-2D materials, and we have implemented this in the CALYPSO package. The new 2D module allows the relaxation of atoms in the perpendicular direction within a predefined range. In order to improve the global structural search efficiency, several techniques including structural similarity determination, symmetry, and distance constraint enforcement and a space gridding in structure generations were developed. The high efficiency of 2D structure search module was illustrated by benchmark studies of known 2D systems including single-layer (carbon, boron, and boron nitride), multi-layer (bilayers and trilayers carbon) structures, and quasi-2D materials $\left(\mathrm{MoS}_{2}, \mathrm{MoSe}_{2}, \mathrm{WS}_{2}\right.$, and $\left.\mathrm{WSe}_{2}\right)$. Also, our method shows that some structures of layered materials are not planar as predicted by a strict 2D structure search. Utilizing this new module in CALYPSO code, we studied the structure of a new family of layered $\mathrm{B}_{\mathrm{x}} \mathrm{N}_{\mathrm{y}}$ compounds. We found that not only the structure, but also the parity of the rings that are the building blocks of these planar materials can be altered by the composition. Through a change in parity, we can tune the band gap of the materials from 2.20 to $5.40 \mathrm{eV}$, 
showing strong potential for applications to electronics and optoelectronics. Overall, our approach provides an efficient and reliable method for searching structures and designing novel 2D and quasi-2D functional materials.

\section{ACKNOWLEDGMENTS}

The authors acknowledge funding support from the National Natural Science Foundation of China (Grant Nos. 11274136, 91022092, and 11025418), the research fund of Key Laboratory of Surface Physics and Chemistry (SPC201103), and the China 973 Program (Grant No. 2011CB808204). Yanchao Wang also acknowledges the support from the CSRC. Maosheng Miao acknowledges support from the ConvEne IGERT Program (NSF-DGE 0801627) and MRL, an NSF funded MRSEC center (DMR-1121053). The calculations were performed in the High Performance Computing Center of Jilin University.

${ }^{1}$ K. Novoselov, A. Geim, S. Morozov, D. Jiang, Y. Zhang, S. Dubonos, I. Grigorieva, and A. Firsov, Science 306, 666 (2004).

${ }^{2}$ K. Novoselov, A. Geim, S. Morozov, D. Jiang, M. I. K. I. V. Grigorieva, S. Dubonos, and A. Firsov, Nature (London) 438, 197 (2005).

${ }^{3}$ A. H. C. Neto, F. Guinea, N. Peres, K. Novoselov, and A. Geim, Rev. Mod. Phys. 81, 109 (2009).

${ }^{4}$ T. Bush, C. R. A. Catlow, and P. Battle, J. Mater. Chem. 5, 1269 (1995).

${ }^{5}$ C. J. Pickard and R. Needs, J. Phys.: Condens. Matter 23, 053201 (2011).

${ }^{6}$ A. R. Oganov and C. W. Glass, J. Chem. Phys. 124, 244704 (2006).

${ }^{7}$ D. Deaven and K. Ho, Phys. Rev. Lett. 75, 288 (1995).

${ }^{8}$ N. Abraham and M. Probert, Phys. Rev. B 73, 224104 (2006).

${ }^{9}$ G. Trimarchi and A. Zunger, Phys. Rev. B 75, 104113 (2007).

${ }^{10}$ S. T. Call, D. Y. Zubarev, and A. I. Boldyrev, J. Comput. Chem. 28, 1177 (2007).

${ }^{11}$ D. J. Wales and H. A. Scheraga, Science 285, 1368 (1999).

${ }^{12}$ S. M. Woodley and R. Catlow, Nature Mater. 7, 937 (2008).

${ }^{13}$ D. C. Lonie and E. Zurek, Comput. Phys. Commun. 182, 372 (2011).

${ }^{14}$ Y. Wang, J. Lv, L. Zhu, and Y. Ma, Phys. Rev. B 82, 094116 (2010).

${ }^{15}$ Y. Wang, J. Lv, L. Zhu, and Y. Ma, Comput. Phys. Commun. 183, 2063 (2012); CALYPSO code is free for academic use. Please register at http://www.calypso.cn.

${ }^{16}$ J. Lv, Y. Wang, L. Zhu, and Y. Ma, Phys. Rev. Lett. 106, 015503 (2011).

${ }^{17}$ L. Zhu, H. Wang, Y. Wang, J. Lv, Y. Ma, Q. Cui, and G. Zou, Phys. Rev. Lett. 106, 145501 (2011).

${ }^{18}$ Y. Wang, H. Liu, J. Lv, L. Zhu, H. Wang, and Y. Ma, Nature Commun. 2, 563 (2011).
${ }^{19}$ L. Zhu, Z. Wang, Y. Wang, G. Zou, H. Mao, and Y. Ma, Proc. Natl. Acad. Sci. U.S.A. 109, 751 (2012).

${ }^{20}$ H. Wang, S. T. John, K. Tanaka, T. Iitaka, and Y. Ma, Proc. Natl. Acad. Sci. U.S.A. 109, 6463 (2012).

${ }^{21}$ J. Lv, Y. Wang, L. Zhu, and Y. Ma, J. Chem. Phys. 137, 084104 (2012).

${ }^{22}$ X. Luo, J. Yang, H. Liu, X. Wu, Y. Wang, Y. Ma, S. H. Wei, X. Gong, and H. Xiang, J. Am. Chem. Soc. 133, 16285 (2011).

${ }^{23}$ H. Xiang, B. Huang, Z. Li, S. Wei, J. Yang, and X. Gong, Phys. Rev. X 2, 011003 (2012).

${ }^{24}$ C. W. Glass, A. R. Oganov, and N. Hansen, Comput. Phys. Commun. 175, 713 (2006).

${ }^{25}$ J. Behler, J. Chem. Phys. 134, 74106 (2011).

${ }^{26}$ J. Behler and M. Parrinello, Phys. Rev. Lett. 98, 146401 (2007).

${ }^{27}$ J. Behler, R. Martoňák, D. Donadio, and M. Parrinello, Phys. Rev. Lett. 100, 185501 (2008).

${ }^{28}$ Particle Swarm Optimization, edited by J. Kennedy and R. Eberhart (IEEE, 1995).

${ }^{29}$ Particle Swarm Optimization: Developments, Applications and Resources, edited by Y. Shi (IEEE, 2001).

${ }^{30}$ A New Optimizer Using Particle Swarm Theory, edited by R. Eberhart and J. Kennedy (IEEE, 1995).

${ }^{31}$ G. Kresse and D. Joubert, Phys. Rev. B 59, 1758 (1999).

${ }^{32}$ G. Kresse and J. Furthmüller, Phys. Rev. B 54, 11169 (1996).

${ }^{33}$ J. M. Soler, E. Artacho, J. D. Gale, A. García, J. Junquera, P. Ordejón, and D. Sánchez-Portal, J. Phys.: Condens. Matter 14, 2745 (2002).

${ }^{34}$ P. Giannozzi et al., J. Phys.: Condens. Matter 21, 395502 (2009).

${ }^{35}$ S. J. Clark, M. D. Segall, C. J. Pickard, P. J. Hasnip, M. I. J. Probert, K. Refson, and M. C. Payne, Z. Kristallogr. 220, 567 (2005).

${ }^{36}$ V. Milman et al., J. Mol. Struct. 954, 22 (2010).

${ }^{37}$ J. D. Gale, J. Chem. Soc., Faraday Trans. 93, 629 (1997).

${ }^{38}$ P. E. Blöchl, Phys. Rev. B 50, 17953 (1994).

${ }^{39}$ J. P. Perdew, K. Burke, and M. Ernzerhof, Phys. Rev. Lett. 77, 3865 (1996).

${ }^{40}$ H. J. Monkhorst and J. D. Pack, Phys. Rev. B 13, 5188 (1976).

${ }^{41}$ A. K. Geim and K. S. Novoselov, Nature Mater. 6, 183 (2007).

${ }^{42}$ A. Nag, K. Raidongia, K. P. S. S. Hembram, R. Datta, U. V. Waghmare, and C. Rao, ACS Nano 4, 1539 (2010).

${ }^{43}$ H. Tang and S. Ismail-Beigi, Phys. Rev. Lett. 99, 115501 (2007).

${ }^{44}$ X. Li, X. Wu, X. C. Zeng, and J. Yang, ACS Nano 6, 4104 (2012).

${ }^{45}$ J. Heyd, G. E. Scuseria, and M. Ernzerhof, J. Chem. Phys. 118, 8207 (2003).

${ }^{46}$ K. F. Mak, J. Shan, and T. F. Heinz, Phys. Rev. Lett. 104, 176404 (2010).

${ }^{47}$ T. Ohta, A. Bostwick, T. Seyller, K. Horn, and E. Rotenberg, Science 313, 951 (2006).

${ }^{48}$ C. A. Diaz Pinto, D. De, V. G. Hadjiev, and H. Peng, ACS Nano 6, 1142 (2012).

${ }^{49}$ Y. Xu, X. Li, and J. Dong, Nanotechnology 21, 065711 (2010).

${ }^{50}$ M. Koshino and E. McCann, Phys. Rev. B 79, 125443 (2009).

${ }^{51}$ J. H. Warner, M. Mukai, and A. I. Kirkland, ACS Nano 6, 5680 (2012).

${ }^{52}$ R. Wold, Phys. Rev. B 35, 6195 (1987). 
The Journal of Chemical Physics is copyrighted by the American Institute of Physics (AIP). Redistribution of journal material is subject to the AIP online journal license and/or AIP copyright. For more information, see http://ojps.aip.org/jcpo/jcper/jsp 